Pensamiento Crítico N. ${ }^{\circ}$ 15, pp. 139-146

\title{
Política monetaria y la tasa de interés de las grandes empresas
}

\author{
Richard Roca Garay ${ }^{1}$
}

\section{RESUMEN}

El trabajo analiza el comportamiento del costo de crédito de las mejores empresas para el periodo 2003-2011, el cual que se conoce como tasa de interés corporativa, y encuentra que es determinado básicamente por la tasa de referencia que fija cada mes el Banco Central de Reserva del Perú.

Palabras Claves: Política monetaria, tasas de interés de referencia, tasa preferencial, tasa corporativa, costo del crédito.

\section{ABSTRACT}

This work analyze the behavior of cost of credit of best enterprises for the period 2003-2011 which is known as corporate rate and is determined basically by the interest rate that the Central Bank fix each month in Peru.

Keywords: Monetary Policy, Interest rate of reference, Prime rate, Corporate rate, cost of credit.

$\overline{1}$ Profesor Principal de Economía de la UNMSM. Email: rhroca@yahoo.com 


\section{Pensamiento Crítico N. 15}

\section{Introducción}

La tasa de interés es uno de los precios más importantes de cualquier economía. El costo del crédito no es el mismo para todos los agentes económicos. En cualquier momento del tiempo en cualquier economía existe una amplia variedad de tasas de interés dependiendo de una serie de factores entre ellas los diferentes niveles de riesgo crediticio de cada prestatario. Las mejores empresas, las que tienen mejor historial financiero y generan más confianza, ofrecen mayores garantías de repago suelen pagar tasas de interés más bajas en el sistema bancario a las que se le denomina la tasa preferencial o tasa corporativa.

El estudio analiza el grado de influencia de la tasa de referencia del Banco Central de Reserva del Perú sobre el comportamiento de la tasa corporativa.

Erick, Lahura (2005) usando la tasa interbancaria como instrumento de política monetaria analiza la influencia de la política monetaria en el Perú para el 1995-2004. Encuentra que el traspaso de largo plazo es aún incompleto pero que se ha incrementado luego del anuncio del corredor de tasas de interés de referencia y se ha reforzado con la adopción del esquema de metas explícitas de inflación desde el 2002. Además que el anuncio del corredor de tasas de interés ha incrementado la velocidad de ajuste de las tasas de mercado ante cambios en la tasa interbancaria; y que en el corto plazo, las tasas de interés de mercado responden asimétricamente cuando la tasa de interés interbancaria sube o baja.

\section{Tasas de interés}

La tasa de interés es el costo de oportunidad de no prestar dinero. En ese costo influyen una serie de factores, entre ellos el riesgo de incumplimiento, la tasa de inflación y los costos de captación de fondos. A nivel de una economía hay muchos agentes con diversos niveles de riesgo de incumplimiento por lo que las tasas de interés no son las mismas. 


\section{Richard Roca Garay}

La tasa de referencia es la tasa que el BCRP fija cada mes con el objetivo fundamental de mantener controlada la inflación dentro del rango meta de 1 a 3\% anualizado. Pero al fijar dicha tasa influye en la determinación de algunas tasas de interés como la Tasa Activa en Moneda Nacional (TAMN), la Tasa Corporativa (TCor). En la Figura 1 también se muestra la evolución de la Tasa Activa en Moneda Extranjera (TAMEX).

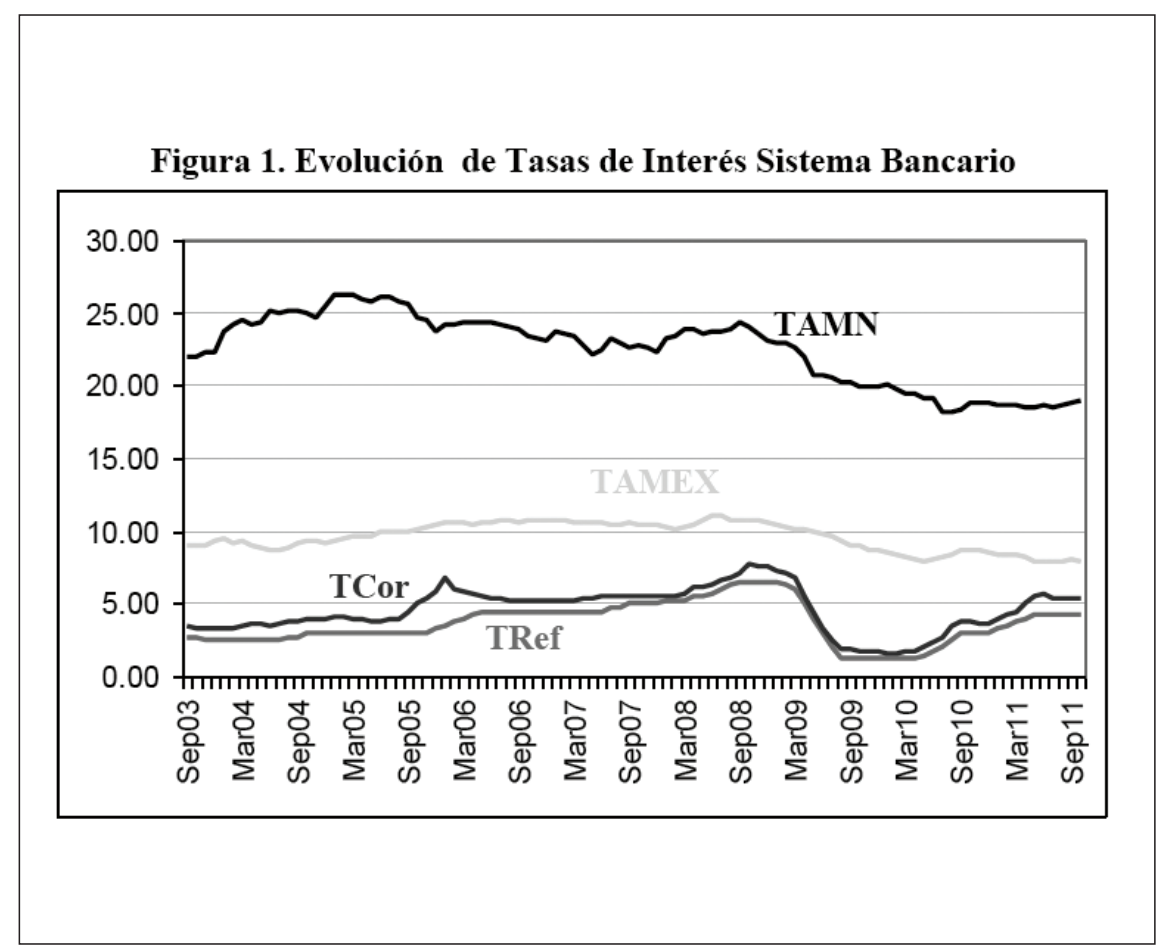

Como señalan Rossini y Vega (2007) el BCRP al fijar la tasa de interés de referencia (TRef) controla las presiones inflacionarias a través de varios canales de transmisión. Como se muestra en la Figura 2. 


\section{Pensamiento Crítico N. IS}

Figura 2. Mecanismo de Transmisión de la Política Monetaria en el Perú

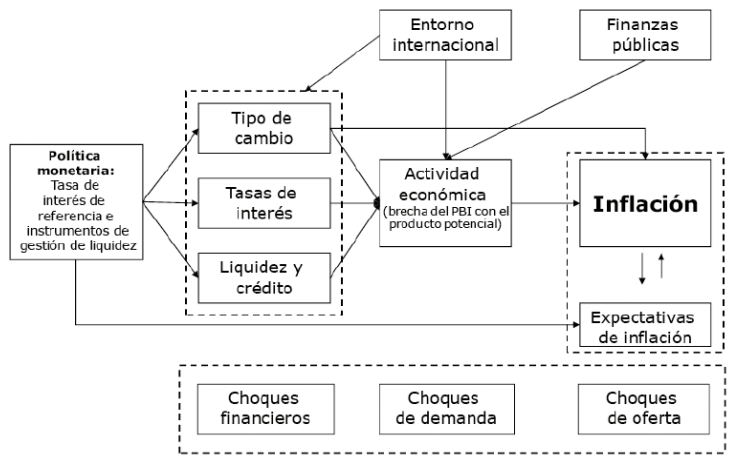

Simultáneamente el BCRP fija las tasas de regulación (TReg) y la Tasa Overnight (TOve) que son las tasas que el BCRP cobra por presta a los bancos y que paga por depósitos de los bancos en el BCRP, respectivamente las cuales se muestra que evolucionan paralelamente a la tasa de referencia como se observa en la Figura 3.

Figura 3. Tasa de interés de Referencia, Corporativa, de Regulación y Overnight

(\% anual. Set2003 - set2011)

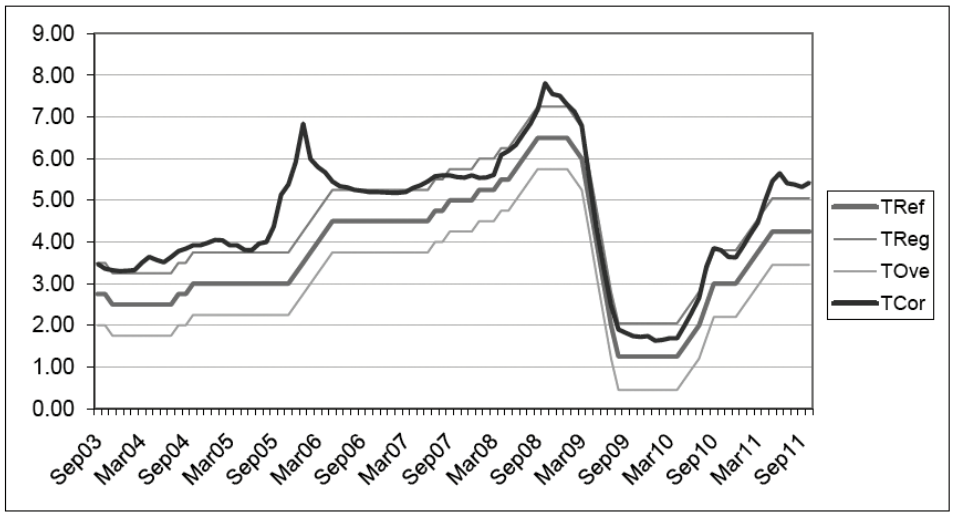




\section{Richard Roca Garay}

En la figura 3 se observa que en algunos meses entre setiembre del 2007 y abril del 2008, además desde abril del 2009 hasta marzo del 2011 la tasa corporativa estuvo por debajo de la tasa de regulación monetaria debido posiblemente a exceso de liquidez.

La evolución de las tasas de interés de referencia y tasa corporativa sugiere que la tasa de referencia influye directa y decisivamente a la tasa corporativa.

\section{Análisis empírico}

Usando datos mensuales se prueba la relación lineal entre la tasa de interés corporativa y la tasa de referencia:

$$
\text { TCor }=b_{0}+b_{1} \text { TRef }
$$

Al correr una regresión lineal $\mathrm{MCO}$ entre la tasa corporativa (TCor) y la tasa de referencia (TRef) se obtiene el siguiente resultado:

Dependent Variable: TCOR

\section{Cuadro 1.}

Method: Least Squares

Date: 11/21/11 Time: 18:17

Sample(adjusted): 2002:01 2011:10

Included observations: 118 after adjusting endpoints

\begin{tabular}{lrllr}
\hline \hline \multicolumn{1}{c}{ Variable } & Coefficient & Std. Error & t-Statistic & Prob. \\
\hline \hline \multicolumn{1}{c}{ C } & 1.048639 & 0.165484 & 6.336807 & 0.0000 \\
\multicolumn{1}{c}{ TREF } & 0.983772 & 0.043160 & 22.79344 & 0.0000 \\
\hline \hline R-squared & 0.817478 & Mean dependent var & 4.585424 \\
Adjusted R-squared & 0.815905 & S.D. dependent var & 1.456172 \\
S.E. of regression & 0.624790 & Akaike info criterion & 1.914001 \\
Sum squared resid & 45.28203 & Schwarz criterion & 1.960962 \\
Log likelihood & -110.9261 & F-statistic & 519.5407 \\
Durbin-Watson stat & 0.370474 & Prob(F-statistic) & 0.000000 \\
\hline \hline
\end{tabular}




\section{Pensamiento Crítico N. ${ }^{\circ}$}

Figura 4. Ajuste de la regresión estimada.

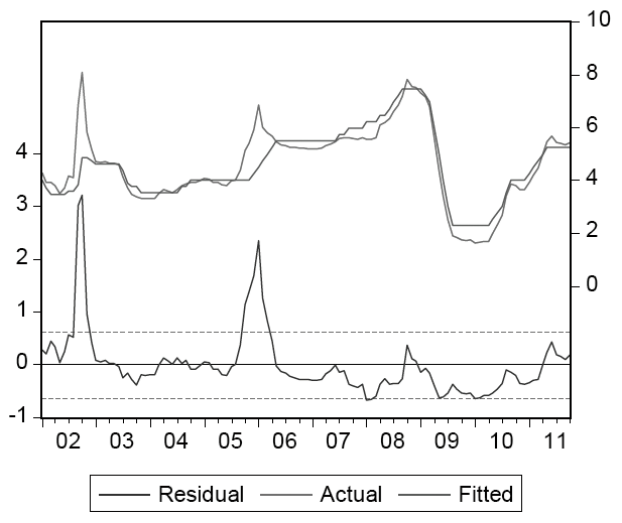

En el Cuadro 1 se observa fuertes problemas de auto correlación (DW=0.370474). Además la Figura 1 muestra la evolución de la tasa corporativa realizada y la estimada. Se nota que el ajuste no es muy bueno.

Al corregir la auto correlación incluyendo procesos autorregresivos $\mathrm{AR}(1)$ y $\mathrm{AR}(2)$ mejora sensiblemente la regresión como se muestra en el siguiente cuadro:

\section{Cuadro 2.}

Dependent Variable: TCor

Method: Least Squares

Date: 11/21/11 Time: 18:44

Sample(adjusted): 2002:03 2011:10

Included observations: 116 after adjusting endpoints

Convergence achieved after 9 iterations

\begin{tabular}{lrllr}
\hline \hline \multicolumn{1}{c}{ Variable } & Coefficient & Std. Error & t-Statistic & Prob. \\
\hline C & 0.961259 & 0.373873 & 2.571082 & 0.0114 \\
TRef & 1.007094 & 0.094806 & 10.62267 & 0.0000 \\
AR(1) & 1.018743 & 0.092793 & 10.97865 & 0.0000 \\
AR(2) & -0.249532 & 0.091999 & -2.712345 & 0.0077 \\
\hline \hline R-squared & 0.942489 & Mean dependent var & 4.593966 \\
Adjusted R-squared & 0.940949 & S.D. dependent var & 1.467087 \\
S.E. of regression & 0.356508 & Akaike info criterion & 0.808956 \\
Sum squared resid & 14.23499 & Schwarz criterion & 0.903907 \\
Log likelihood & -42.91942 & F-statistic & 611.8227 \\
Durbin-Watson stat & 1.926463 & Prob(F-statistic) & 0.000000 \\
\hline \hline Inverted AR Roots & .61 & .41 & & \\
\hline \hline
\end{tabular}




\section{Richard Roca Garay}

\section{Figura 5. Ajuste de la regresión corregida de autorrelación}

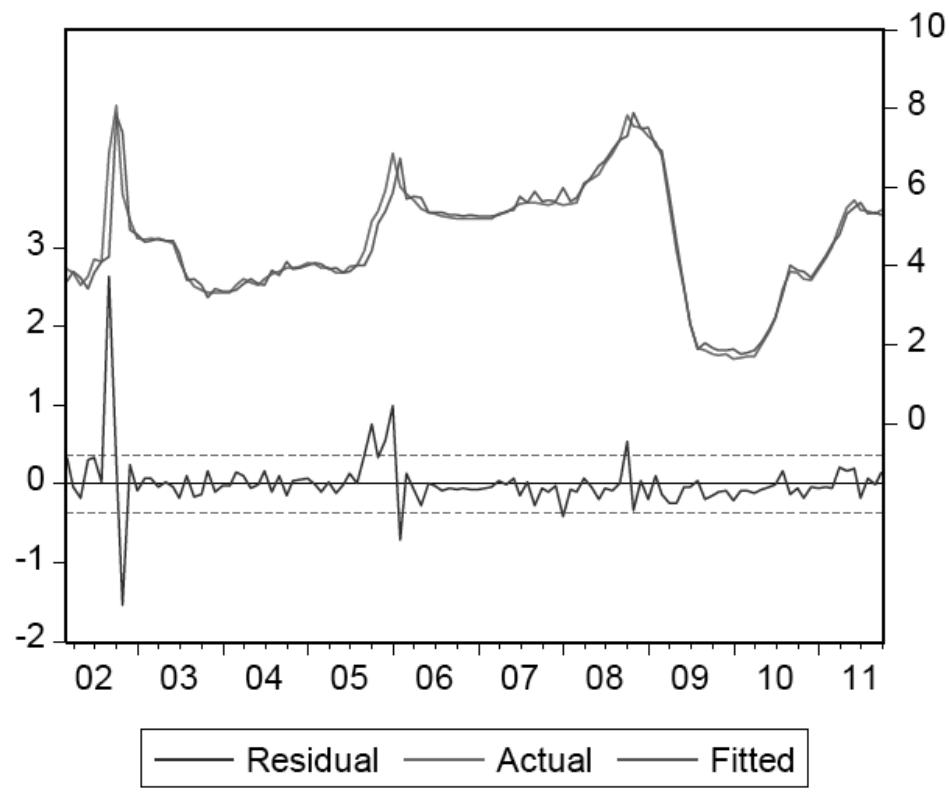

Se observa que se resuelve el problema de auto correlación, la significancia estadística de la tasa de referencia es alta $(\mathrm{t}=10.62267)$ y un $\mathrm{R}^{2}$ alto $(0.942489)$ y además muestra que la influencia de la tasa de referencia en al tasa corporativa es fuerte: 1007094

\section{Conclusiones}

El resultado de las regresiones mostrada en el Cuadro 2 implica que el BCRP influye decisivamente en el costo del crédito de las grandes empresas, sobre todo de las que tienen acceso a la tasa preferencial ya que el valor de coeficiente de la tasa de referencia es alta $\left(b_{1}=1007094\right)$. 


\section{Pensamiento Crítico N. ${ }^{\circ}$}

\section{Bibliografía}

ESPINOZA, Marco (2003). Traspaso de la tasa de interés de la banca comercial. ¿Es Chile Atípico? BCCh dtbc221.

LAHURA, Erick (2005). El efecto traspaso de la tasa interés y la política monetaria en el perú: 1995 - 2004. Documentos de Trabajo 244. Departamento de economía. Pontificia Universidad Católica del Perú.

ROSSINI, Renzo y Marco, Vega (2007). "El mecanismo de transmisión de la política monetaria en un entorno de dolarización financiera: El caso del Perú entre 1996 y 2006". Revista Estudios Económicos N. ${ }^{\circ}$ 17. Banco Central de Reserva del Perú. 